Marcel A. Kopp, MD*

Ralf Watzlawick, MD*

Peter Martus, PhD*

Vieri Failli, PhD

Felix W. Finkenstaedt, MD

Yuying Chen, MD, PhD

Michael J. DeVivo, DrPH

Ulrich Dirnagl, MD

Jan M. Schwab, MD,

$\mathrm{PhD}$

Correspondence to

Dr. Schwab:

Jan.Schwab@osumc.edu
Supplemental data at Neurology.org

\section{Long-term functional outcome in patients with acquired infections after acute spinal cord injury}

\section{ABSTRACT}

Objective: To investigate whether prevalent hospital-acquired pneumonia and wound infection affect the clinical long-term outcome after acute traumatic spinal cord injury (SCI).

Methods: This was a longitudinal cohort study within the prospective multicenter National Spinal Cord Injury Database (Birmingham, Alabama). We screened datasets of 3,834 patients enrolled in 20 trial centers from 1995 to 2005 followed up until 2016. Eligibility criteria were cervical SCI and American Spinal Cord Injury Association impairment scale A, B, and C. Pneumonia or postoperative wound infections $(\mathrm{Pn} / \mathrm{Wi})$ acquired during acute medical care/inpatient rehabilitation were analyzed for their association with changes in the motor items of the Functional Independence Measure ( $F I M_{\text {motor }}$ ) using regression models (primary endpoint 5-year follow-up). Pn/Wi-related mortality was assessed as a secondary endpoint (10-year follow-up).

Results: A total of 1,203 patients met the eligibility criteria. During hospitalization, 564 patients $(47 \%)$ developed Pn/Wi (pneumonia $n=540$; postoperative wound infection $n=11$; pneumonia and postoperative wound infection $n=13$ ). Adjusted linear mixed models after multiple imputation revealed that $\mathrm{Pn} / \mathrm{Wi}$ are significantly associated with lower gain in $\mathrm{FIM}$ motor up to 5 years after $\mathrm{SCl}(-7.4$ points, $95 \%$ confidence interval $[\mathrm{Cl}]-11.5$ to -3.3$)$. Adjusted Cox regression identified $\mathrm{Pn} / \mathrm{Wi}$ as a highly significant risk factor for death up to 10 years after SCI (hazard ratio 1.65 , 95\% $\mathrm{Cl} 1.26$ to 2.16$)$.

Conclusion: Hospital-acquired $\mathrm{Pn} / \mathrm{Wi}$ are predictive of propagated disability and mortality after $\mathrm{SCl}$. Pn/Wi qualify as a potent and targetable outcome-modifying factor. Pn/Wi prevention constitutes a viable strategy to protect functional recovery and reduce mortality. Pn/Wi can be considered as rehabilitation confounders in clinical trials. Neurology ${ }^{\circledR}$ 2017;88:892-900

\section{GLOSSARY}

AIS = American Spinal Cord Injury Association impairment scale; ASIA = American Spinal Cord Injury Association; $\mathbf{F I M}_{\text {motor }}=$ motor items of the Functional Independence Measure; ISNCSCI = International Standards for Neurologic Classification of Spinal Cord Injury; NSCID = National Spinal Cord Injury Database; $\mathbf{P n} / \mathbf{W i}=$ pneumonia or postoperative wound infection; $\mathbf{S C l}=$ spinal cord injury.

Despite increasing translational efforts for developing new treatments for spinal cord injury (SCI), ${ }^{1,2}$ a neurobiological remedy has not yet been established. ${ }^{3,4}$ Moreover, the rate of endogenous recovery can vary substantially regardless of similar lesion size and location, implying the existence of outcome-modifying factors after SCI. Acquired respiratory infections $s^{5,6}$ are the main cause of death after $\mathrm{SCI}^{7}$ and the consecutively reduced life expectancy in patients with chronic SCI has been refractory to improvement over the last 3 decades. ${ }^{8}$

The loss of CNS control over the peripheral immune system results in a SCI-induced immunodeficiency syndrome, ${ }^{9-17}$ which fosters susceptibility to SCI-associated infections. ${ }^{18}$ Besides increasing mortality, acquired infections are associated with impaired neurologic recovery within

\footnotetext{
*These authors contributed equally to this work.

From the Department of Neurology and Experimental Neurology, Clinical and Experimental Spinal Cord Injury Research (Neuroparaplegiology) (M.A.K., R.W., V.F., F.W.F., J.M.S.), and Center for Stroke Research Berlin (U.D.), Charité-Universitätsmedizin Berlin; Department of Clinical Epidemiology and Applied Biostatistics (P.M.), Eberhard Karls Universität Tübingen, Germany; National Spinal Cord Injury Statistical Center (Y.C., M.J.D.), Department of Physical Medicine and Rehabilitation, University of Alabama at Birmingham; Paraplegiology (Spinal Cord Injury Division), Department of Neurology (J.M.S.), and Departments of Neuroscience and Center for Brain and Spinal Cord Repair and Physical Medicine and Rehabilitation, The Neurological Institute (J.M.S.), The Ohio State University, Wexner Medical Center, Columbus. Go to Neurology.org for full disclosures. Funding information and disclosures deemed relevant by the authors, if any, are provided at the end of the article.
} 
the first year after SCI, as we demonstrated in a previous study. ${ }^{19}$ However, the long-term effect of hospital-acquired infections on disability and mortality after SCI remains elusive.

Within a large prospective, multicenter, observational database, ${ }^{20-23}$ this cohort study investigates whether infections with onset during the initial hospitalization period restrict the patients' recovery of physical independence. In particular, hospital-acquired pneumonia or postoperative wound infection $(\mathrm{Pn} / \mathrm{Wi})$ are analyzed. Functional recovery during 5-year follow-up is measured with the motor items of the Functional Independence Measure $\left(\mathrm{FIM}_{\text {motor }}\right)$ as the most widely accepted and applied instrument for comprehensive assessment of rehabilitation outcome. ${ }^{24}$ Considering $\mathrm{Pn} / \mathrm{Wi}$-related deaths as a putative cause of loss to follow-up, we analyze the infectionassociated mortality during a period of 10 years post SCI.

METHODS Database information. The datasets were obtained from the prospective multicenter National Spinal Cord Injury Database (NSCID), Birmingham, Alabama. Data of patients with acute traumatic SCI were collected in specialized SCI care centers (SCI model systems). Various procedures to assure quality and validity of collected data have been described in detail in the syllabus and in previous publications. ${ }^{20-23}$

Standard protocol approvals, registrations, and patient consents. The participating institutions' Review Boards approved the database and enrollment was performed in accordance with the Declaration of Helsinki. The relevant data collection syllabuses 2000-2005 and 2006-2011 as well as the case report forms are available on the NSCID website (nscisc.uab.edu/ nscisc-database.aspx). All participants were informed about the aim of the study and gave their written informed consent.

Eligibility criteria. The process of dataset selection according to predefined criteria is illustrated in the enrollment chart (figure 1A). Patients with acute traumatic SCI were assigned to longitudinal follow-up according to the NSCID condition of admission within 24 hours after injury. Datasets from patients enrolled from January 1995 to December 2005 were screened. This time period was determined by the NSCID data collection protocol: (1) long-term follow-up of the $\mathrm{FIM}_{\text {motor }}$ as primary outcome parameter was implemented in 1995 and (2) $\mathrm{Pn} / \mathrm{Wi}$ as clinical key parameters of the study were removed from the data collection protocol in 2005.

Patients with cervical SCI and clear motor impairment as defined by the American Spinal Cord Injury Association (ASIA) impairment scale (AIS) grade A, B, or C were selected for the analysis. The study population was confined to cervical SCI and to AIS A-C severity in order to limit the otherwise huge heterogeneity of the functional baseline after acute SCI and to represent a relevant clinical cohort for interventional testing. Furthermore, the selection of patients with cervical SCI with a clear motor deficit prevents ceiling effects in the functional long-term outcome measures most relevant for patients with AIS D. ${ }^{3,25}$
Exclusion criteria were age $<16$ or $>75$ years, stay in hospital or nursing home prior to injury, or rehospitalization for unspecified infectious and parasitic diseases during follow-up. Furthermore, datasets with missing neurologic or functional baseline examination at admission to acute medical care and to inpatient rehabilitation were excluded. The AIS is also encoded as missing if important medical conditions prevent International Standards for Neurologic Classification of Spinal Cord Injury (ISNCSCI) examination. Thus, serious concomitant injuries affecting the level of consciousness such as severe and moderate traumatic brain injury are excluded. In addition, datasets without any information on Pn/Wi during the entire period of initial hospitalization were excluded (figure 1A).

Assessments. The FIM $_{\text {motor }}$ assesses the degree of disability by measuring the most common functional meaningful actions of daily life (figure e-1 at Neurology.org) and correlates very well with the SCI-specific Spinal Cord Independence Measure. ${ }^{26}$ $\mathrm{FIM}_{\text {motor }}$ data were collected using observer's ratings or structured interviews as recommended by its developers. ${ }^{27}$ The FIM $_{\text {motor }}$ items were assessed at 4 subsequent time points during a follow-up period of 5 years: at admission to inpatient rehabilitation (baseline), at discharge from inpatient rehabilitation (discharge), at the first annual examination after SCI (1 year), and at the 5-year examination (5 years) after SCI (figure 1B). For patient classification according to the ISNCSCI, ${ }^{28}$ the injury severity (AIS) and the neurologic level were assessed at admission to acute care or inpatient rehabilitation. Furthermore, sociodemographic and clinical baseline characteristics were collected.

Specific hospital-acquired infections documented in the NSCID are pneumonia and postoperative wound infections. Pneumonia is defined as a state of lung tissue inflammation of infectious etiology with radiographic demonstration of parenchymal disease. Postoperative wound infection is defined as wound infection occurring at the site of spinal surgery in reasonable relation to the date of surgery. The onset of both types of infection must have been during acute medical or surgical care or inpatient rehabilitation. Mortality of the study participants was assessed using information on the date of death, which is collected through various mechanisms such as clinical records, obituary, returned mail, and regularly performed social security death index queries.

Data processing. NSCID data are stored in 2 different sequences, form I variables (initial treatment up to discharge from inpatient rehabilitation) and form II variables (long-term follow-up), which were extracted by database request in August 2009 and subsequently updated in October 2012 and merged into a MySQL Database (version 5.5.8; Oracle Corporation, Redwood City, CA) operating the programming language PHP (version 5.3.4; The PHP Group).

For further data processing, the MySQL database was imported into an SPSS (Chicago, IL) data file. Pneumonia or postoperative wound infection documented at least once during initial hospital care were combined into one variable for analysis $(\mathrm{Pn} / \mathrm{Wi})$. From the $13 \mathrm{FIM}_{\text {motor }}$ items, the $\mathrm{FIM}_{\text {motor }}$ total score and 4 different FIM $_{\text {motor }}$ subscores were calculated. Differences between FIM $_{\text {motor }}$ at baseline and the follow-up assessments were calculated. The single neurologic level was calculated from the sidespecific sensory and motor levels according to the ISNCSCI ${ }^{28}$ definitions. Neurologic level and AIS missing at admission to inpatient rehabilitation were replaced by values collected at inclusion in the NSCID.

Data on survival as of January 2016 were included into the dataset. The survival was calculated as the time from date of injury to date of death, censoring at 10 years after SCI at latest. 
Figure 1 Dataset selection and analysis chart

A

Datasets of patients enrolled into the longitudinal NSCID from 1995 to 2005 and followed up for 5 years $(\mathrm{N}=3,834)$

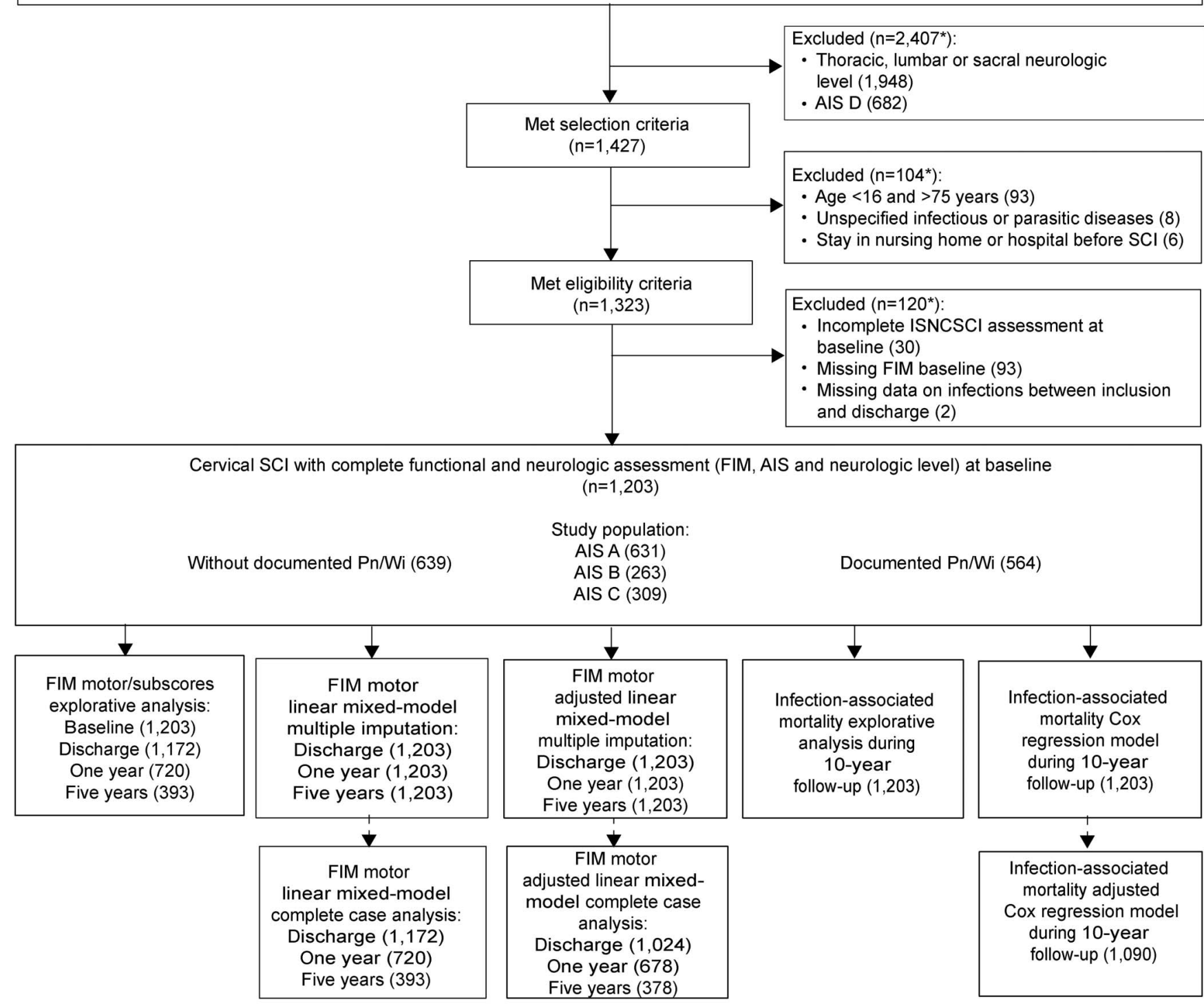

B

FIM motor assessment points following spinal cord injury

Admission to rehabilitation (baseline): 17 (9-31) days

Discharge from inpatient rehabilitation: 87 (61-122) days

One-year examination: $13(12-15)$ months

Five-year examination: 62 (60-65) months

(A) Patients enrolled into the National Spinal Cord Injury Database (NSCID) within 1 day after spinal cord injury (SCI) were assigned to long-term follow-up. Datasets from the NSCID collected between 1995 and 2005 comprise data on pneumonia or postoperative wound infection (Pn/Wi) and motor items of the Functional Independence Measure (FIM) long-term outcome. Thus, those datasets were screened for eligibility. The data for statistical analysis were selected stepwise with respect to quality aspects of the particular assessment tools, possible confounding factors, and the availability of key baseline data. The linear mixed regression models were performed as a complete case analysis and-in order to control for attrition bias-after multiple imputation. *Total numbers may differ from subgroup numbers because some items applied multiple times. (B) Assessment points following $\mathrm{SCl}$ are indicated representing medians and interquartile range. AIS = American Spinal Cord Injury Association impairment scale; ISNCSCI = International Standards for Neurologic Classification of Spinal Cord Injury.

Statistical analysis. The groups for statistical comparison were defined as patients with $\mathrm{Pn} / \mathrm{Wi}$ vs patients without $\mathrm{Pn} / \mathrm{Wi}$. The distribution of continuous variables was described as median and quartiles. The Mann-Whitney test was applied to compare between the groups. Categorical variables were reported as frequencies and percentages and compared using the $\chi^{2}$ test. Survival curves were drawn using the Kaplan-Meier method. All analyses were performed for the total study population and the AIS subgroups.

In order to correct for attrition bias, linear mixed regression models were performed after multiple imputation. Multiple 
Table 1 Baseline characteristics and clinical data

$\begin{array}{lllc}\text { Baseline characteristics } & \text { Without Pn/Wi } & \text { Pn/Wi } & p \text { Value } \\ \text { Age, y, median (IQR) } & 35(23-48) & 34(24-48) & 0.92 \\ \text { Male sex, n/total n (\%) } & 494 / 639(77.3) & 470 / 564(83.3) & 0.009 \\ \text { Caucasian, n/total n (\%) } & 401 / 612(65.5) & 363 / 548(66.2) & 0.80 \\ \text { Working, n/total n (\%) } & 440 / 587(75.0) & 363 / 503(72.2) & 0.30 \\ \text { High school or university, n/total n (\%) } & 412 / 578(71.3) & 321 / 480(66.9) & 0.12 \\ \text { AIS A, n/total n (\%) } & 261 / 639(40.8) & 370 / 564(65.6) & <0.001 \\ \text { AIS B, n/total n (\%) } & 157 / 639(24.6) & 106 / 564(18.8) & \\ \text { AIS C, n/total n (\%) } & 221 / 639(34.6) & 88 / 564(15.6) & \\ \text { Neurologic level C1-C4, n/total n (\%) } & 266 / 639(41.6) & 329 / 564(58.3) & <0.001 \\ \text { Neurologic level C5-C8, n/total n (\%) } & 373 / 639(58.4) & 235 / 564(41.7) & \\ \text { FIM } \text { motor, median (IQR) } & 13(13-20) & 13(13-14.75) & <0.001\end{array}$

Abbreviations: AIS $=$ American Spinal Cord Injury Association impairment scale; $\mathrm{FIM}_{\text {motor }}=$ motor items of the Functional Independence Measure; IQR = interquartile range; $\mathrm{Pn} / \mathrm{Wi}=$ pneumonia or postoperative wound infection.

The Mann-Whitney test was applied for age and FIM motor; the $\chi^{2}$ test was used for the other variables.
RESULTS Dataset characteristics and clinical data. Datasets of 3,834 patients with SCI were enrolled in the longitudinal NSCID database between 1995 and 2005 (figure 1A). After application of the eligibility criteria, 1,203 datasets from patients with cervical SCI of AIS grades A, B, and C recruited in 20 SCI centers between January 1995 and December 2005 were included in the analysis. The FIM $_{\text {motor }}$ was followed up for 5 years after SCI until July 2011 and the survival status up to 10 years after SCI until January 2016.

During acute medical and rehabilitative inpatient care, 564 patients (47\%) developed Pn/Wi. Within this group, 540 are classified as pneumonia, 11 as postoperative wound infection, and 13 as pneumonia and postoperative wound infection.

The baseline characteristics of patients with or without $\mathrm{Pn} / \mathrm{Wi}$ are similar in terms of age, ethnic group, working status, and educational level (table 1). The groups reveal a statistical difference for the following characteristics: sex, AIS, neurologic level, and FIM at baseline, particularly with higher and more severe lesions in the group with $\mathrm{Pn} / \mathrm{Wi}$ (table 1).

imputation $^{29,30}$ was regression-based and performed in the analysis dataset $(n=1,203$, figure $1 \mathrm{~A})$. $\mathrm{FIM}_{\text {motor }}$ at different time points was imputed using linear regression. Ethnic group, marital status, working status, and educational level were imputed using logistic regression. Pn/Wi, age, sex, AIS, neurologic level, and $\mathrm{FIM}_{\text {motor }}$ at baseline were used as predictors in the missing model but not imputed as they had no missing values. The imputation sequence was $\mathrm{Pn} / \mathrm{Wi}$, age, sex, ethnic group, marital status, working status, educational level, AIS at baseline, neurologic level at baseline, FIM motor at baseline, FIM motor at discharge, FIM $_{\text {motor }}$ at 1 year, FIM motor at 5 years. Twenty-five imputations were performed and standard errors, including the variation between the imputation steps, were obtained from the SPSS module. A sensitivity analysis was done for cases with complete data (complete case analysis). The linear mixed regression models were calculated with random intercept and FIM $_{\text {motor }}$ as dependent variable, 4 time points (baseline, discharge, 1-year follow-up, 5-year follow-up) with time vs $\mathrm{Pn} / \mathrm{Wi}$ interaction as primary parameter of interest, and baseline as reference category. The models were adjusted for FIM $_{\text {motor }}$ score at baseline and were sequentially adjusted and stratified for AIS and further adjusted for neurologic level, age, ethnic group, and working status. For the tests of a time vs $\mathrm{Pn} / \mathrm{Wi}$ interaction after multiple imputation, the pooled parameter vectors and the within and between variation covariance matrices were transferred from the SPSS output into an R script to perform a multivariate Wald test as described in the textbook by van Buuren, ${ }^{30}$ section 6.3.1.

For survival analysis, Cox regression models were adjusted and stratified for injury severity (AIS) and subsequently adjusted for neurologic level, age, working status, and educational level.

All tests were 2-sided and the level of significance was 0.05 . Correction for multiple comparison was applied using the Bonferroni method. Data processing and statistical analyses were performed with SPSS Statistics for Windows (version 22.0; IBM, Armonk, NY) and RStudio (version 3.3.0) using syntax files that allow for an exact reproduction of the variable transformation, dataset selection, and analysis based on the merged dataset. Graphs were drawn using SPSS and Prism for Macintosh (version 5.0; GraphPad Software, La Jolla, CA).

\section{Differential functional outcome after SCI measured} by FIM $_{\text {motor }}$ items. The association of $\mathrm{Pn} / \mathrm{Wi}$ with FIM $_{\text {motor }}$ recovery was evaluated at 3 different followup time points (figure 1B) up to 5 years post SCI.

Patients without Pn/Wi have significantly higher differences from baseline in FIM motor total score compared to patients with $\mathrm{Pn} / \mathrm{Wi}$ at discharge (figure 2). The differences between the groups become larger at 1 year and remained highly significant at 5 years (median [interquartile range] control 41 [15.563] vs Pn/Wi 18.5 [9-48.25]). Stratification for AIS reveals clearly significant intergroup differences for AIS A up to 5 years (control 32 [10-51] vs Pn/Wi 15 [5-28.25]). The groups without and with $\mathrm{Pn} / \mathrm{Wi}$ are significantly different at discharge also in the AIS B (control 24 [11.5-40] vs Pn/Wi 14.5 [5.2528.75]) and AIS C strata (control 36 [20-49] vs Pn/Wi 25.5 [8-39]). During further follow-up, no statistically significant intergroup differences were observed in the AIS B and C subgroups (figure 2). The FIM motor subscore analysis demonstrates a recovery pattern comparable to the $\mathrm{FIM}_{\text {motor }}$ total score evaluation in all groups (figure e-2).

The baseline only adjusted linear mixed regression model with the FIM motor $_{\text {total score as a dependent }}$ variable calculated in the total sample reveals a highly significant association between $\mathrm{Pn} / \mathrm{Wi}$ and impaired FIM $_{\text {motor }}$ recovery at all follow-up time points (table e-1) after multiple imputation up to 5 years $(-16.2$ points, $95 \%$ confidence interval $[\mathrm{CI}]-20.1$ to -12.3$)$ as well as in the complete case analysis $(-13.4$ points, $95 \%$ CI -16.6 to -10.1$)$. 

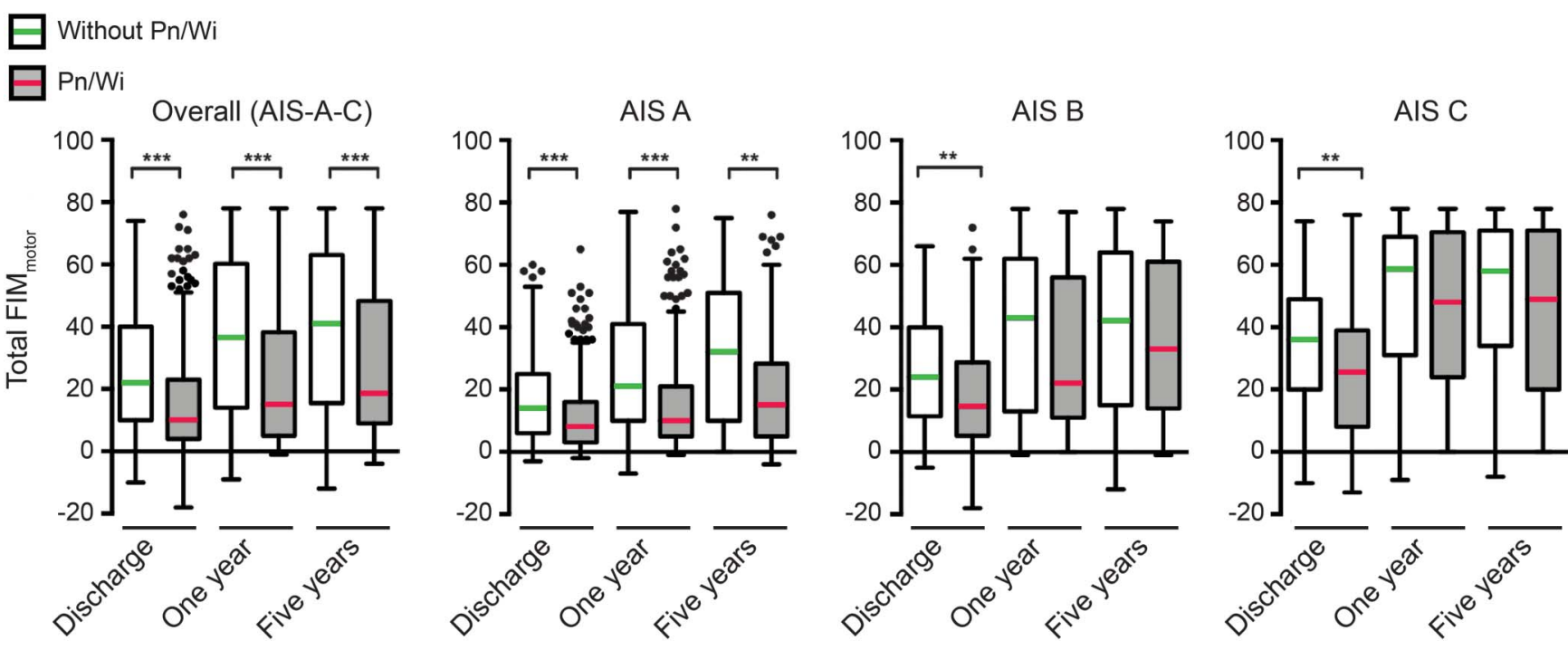

Differences from FIM motor baseline (median) at admission were compared between patients with and without documented pneumonia or postoperative wound infection (Pn/Wi) at discharge, 1 year after spinal cord injury (SCI), and 5 years after SCI. FIM motor was significantly reduced in the Pn/Wi group in the total sample and in AIS A at all time points up to 5 years, whereas in AIS B and C, statistical significance was only transiently present. Sample size: FIM motor score at discharge (d), 1 year, and 5 years: (d) $n=619$ without $P n / W i, n=553$ with Pn/Wi; $(1$ y) $n=398, n=322 ;(5 y) n=217, n=176$. AIS A: (d) $n=251$, $n=363 ;(1 y) n=163, n=205 ;(5 y) n=91, n=118$. AIS B: (d) $n=149, n=104 ;(1 y) n=103, n=61 ;(5 y) n=55, n=35$. AIS C: (d) $n=219, n=86 ;(1$ y) $n=132, n=56 ;(5 y) n=71, n=23$. Boxes are plotted as median and interquartile range; whiskers are defined according to Tukey and outliers are indicated by dots. Mann-Whitney test statistical significances are corrected using the Bonferroni method: ${ }^{*} p<0.05,{ }^{* *} p<0.01, * * * p 0.001$.

The model adjusted for AIS, neurologic level, and sociodemographic variables (table 2) corroborates a clearly significant association of $\mathrm{Pn} / \mathrm{Wi}$ with

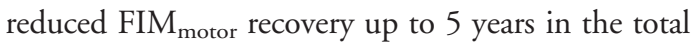
sample after multiple imputation ( -7.4 points, $95 \%$ $\mathrm{CI}-11.5$ to -3.3$)$ and in the complete case analysis $(-5.2$ points, $95 \% \mathrm{CI}-8.4$ to -2.0$)$. In the baseline only adjusted model (table e-1) and at all subsequent steps of adjustment (table 2) time vs $\mathrm{Pn} / \mathrm{Wi}$ interactions are highly significant after multiple imputation and in the complete case analysis.

Stratification of the model for AIS indicates within the AIS A stratum that $\mathrm{Pn} / \mathrm{Wi}$ is associated with significantly impaired FIM motor recovery up to 5 years after multiple imputation and in the complete case analysis. This association reaches statistical significance after multiple imputation in the AIS B stratum at 5 years and a nonsignificant association in AIS C. This is not observable in the complete case analysis (table 2).

Infection-associated mortality. The in-hospital mortality is low in groups with (7 of 564; 1.2\%) and without (2 of $639,0.3 \%) \mathrm{Pn} / \mathrm{Wi}$ and not significantly different between the groups $(p=0.09)$. Long-term survival curves indicate a higher cumulative mortality in the group of patients with $\mathrm{Pn} / \mathrm{Wi}$ compared to patients without $\mathrm{Pn} / \mathrm{Wi}$ in the total population and in the AIS subgroups during the observation period of 10 years (figure 3). The Cox regression revealed that $\mathrm{Pn} / \mathrm{Wi}$ is associated with mortality in the total sample (table e-2).
This was confirmed after adjustment for the AIS (hazard ratio $[95 \%$ CI] 2.08 [1.63-2.66], $p<$ 0.001). Stratification for AIS subgroups demonstrated a Pn/Wi-associated mortality for AIS A and B (table e-2). After subsequent adjustment for AIS, neurologic level, and sociodemographic variables, Cox regression demonstrated a statistically significant association of $\mathrm{Pn} / \mathrm{Wi}$ with mortality in the total sample during 10 years (1.65 [1.26-2.16], $p<0.001)$. In the AIS strata, a statistically significant association was detectable for AIS A patients (table e-3).

DISCUSSION This study provides the first evidence that $\mathrm{Pn} / \mathrm{Wi}$ acquired during initial hospitalization after SCI sustainably affect the recovery of physical independence and long-term survival. Within regression models, adjusted for the most consistent predictors of functional restoration after acute traumatic $\mathrm{SCI},{ }^{31}$ we demonstrate that $\mathrm{Pn} / \mathrm{Wi}$ are a risk factor for poorer gain in the $\mathrm{FIM}_{\text {motor. }}$ This is supported by the results of subgroup analyses, stratified for SCI severity by the AIS. Thus, the complementary assumption that the reduced recovery in FIM $_{\text {motor }}$ is attributable to the baseline differences between the groups with and without $\mathrm{Pn} / \mathrm{Wi}$ is less likely. The observed shrinkage of effect sizes after subsequent model adjustment was expected, because the AIS and the neurologic level are highly predictive of functional recovery. ${ }^{31}$

Moreover, differences in FIM motor $_{\text {recovery between }}$ the groups mainly caused by baseline-dependent 
Table 2 Decrease in motor items of the Functional Independence Measure $\left(\mathrm{FIM}_{\text {motor }}\right)$ associated with infection: Linear mixed model adjusted for neurologic level and sociodemographic covariates

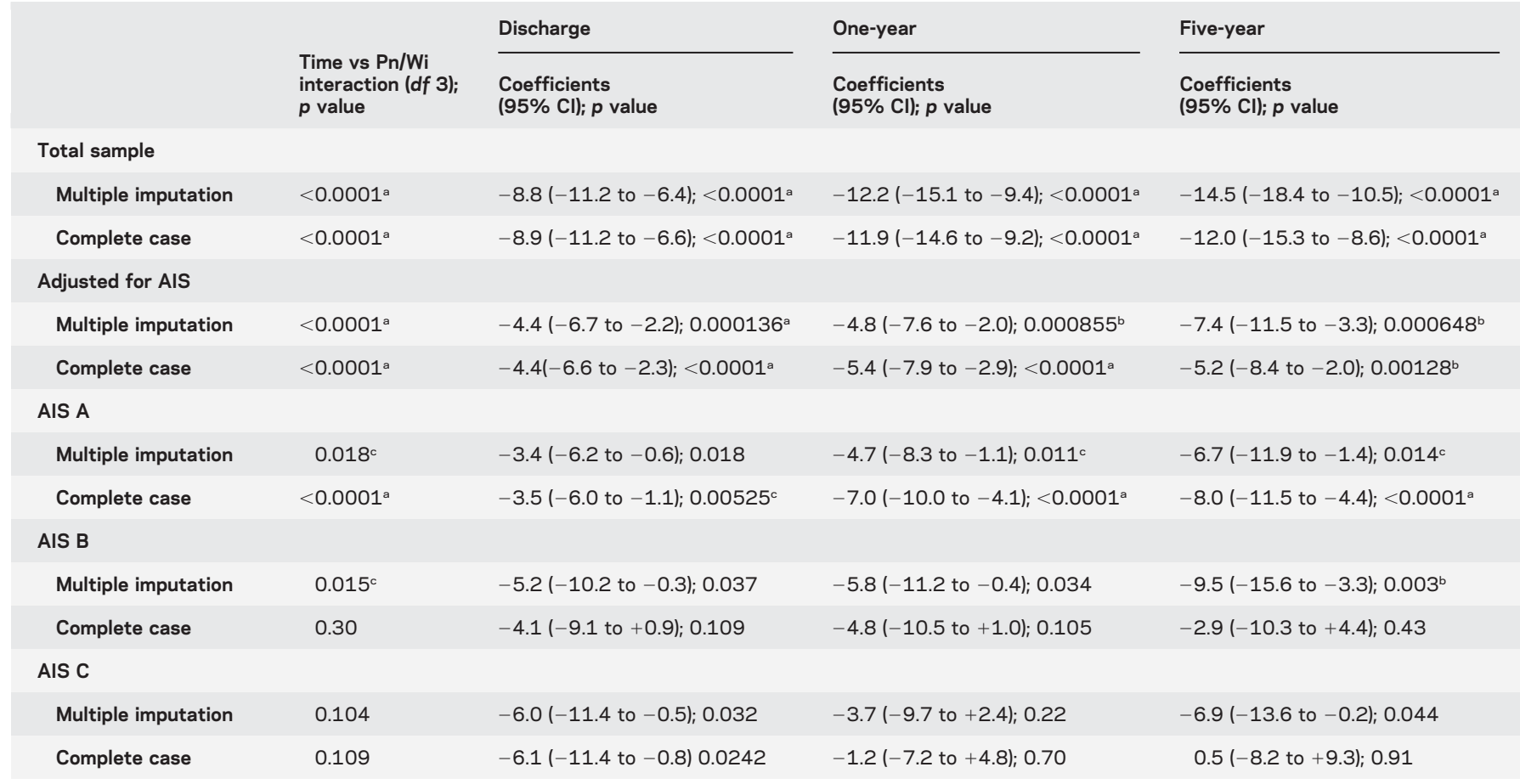

Abbreviations: AIS = American Spinal Cord Injury Association impairment scale; $\mathrm{Cl}=$ confidence interval; $\mathrm{Pn} / \mathrm{Wi}=$ pneumonia and/or postoperative wound infection.

Linear mixed regression model with random intercept and FIM motor as dependent variable, 4 time points (baseline, discharge, 1-year follow-up, 5-year follow-up) with time vs infection interaction as primary parameter of interest, and baseline as reference category, analysis based on multiple imputation (primary analysis) and complete case analysis (sensitivity analysis). Each model was adjusted for sociodemographic variables (age, ethnic group, working status before injury) and neurologic level. The models were calculated without adjustment for AIS (row 1), and subsequently further adjusted (row 2) and stratified for AIS (rows 3-5). In the models using multiple imputation, relative efficiency was larger than 0.97, indicating a sufficient number of imputations $(n=25)$. The fraction of missing information was between 0.008 and 0.031 for discharge, $0.169-0.403$ for 1 -year follow-up, and 0.383-0.714 for 5-year follow-up. Thus for 5-year follow-up results depend strongly on the method of imputation (van Buuren ${ }^{30(p 42)}$ ). Statistical significances are corrected using the Bonferroni method: a $p<0.001 ;{ }^{b} p<0.01 ;{ }^{c} p<0.05$.

compensation $^{3}$ can be ruled out through linking the results of this study on FIM $_{\text {motor }}$ recovery to the results of a previous study on neurologic outcome, particularly in the ASIA motor score. The differences in $\mathrm{FIM}_{\text {motor }}$ recovery between patients with and without $\mathrm{Pn} / \mathrm{Wi}$ detected in this study are in line with published evidence demonstrating impaired ASIA motor score recovery in patients with $\mathrm{Pn} / \mathrm{Wi}$ compared to patients without $\mathrm{Pn} / \mathrm{Wi}$ in the previous study. ${ }^{19}$ Despite not being population-based, the epidemiologic data and endogenous recovery profiles documented in the NSCID are consistent with other large SCI-specific datasets ${ }^{3,4,32}$ and represent the best evidence available to identify disease-modifying factors in SCI at present. The limitation of attrition bias due to loss to follow-up was addressed by multiple imputation, which has been recommended as being more valid than dropping incomplete cases. ${ }^{33}$ The results of the fully adjusted linear mixed regression model after multiple imputation are in line with those of the complete case analysis in the total sample during the complete follow-up period. Stratification of the regression models for AIS revealed an attrition bias in the strata of incomplete
SCI (AIS B and C). This is irrelevant for the stratum of complete SCI (AIS A). Here, multiple imputation and complete case analysis are matching. In patients with incomplete SCI, the association of $\mathrm{Pn} / \mathrm{Wi}$ with

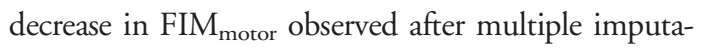
tion at 5 years is not detectable in the complete case analysis. The higher variation of outcome ${ }^{4}$ and the lower sample size in the AIS B and C strata make these groups more susceptible to attrition bias. This is probably also related to increased loss to follow-up due to mortality of more severely affected patients in the group with $\mathrm{Pn} / \mathrm{Wi}$ (figure 3). The explorative survival analyses of the AIS subgroups indicate a nonsignificant association of $\mathrm{Pn} / \mathrm{Wi}$ with mortality also in patients AIS B and C.

$\mathrm{Pn} / \mathrm{Wi}$ is associated with a loss of approximately 7 points in the 5-year follow-up FIM motor, which can, for example, make the difference between no assistance and full assistance in one of the 13 FIM $_{\text {motor }}$ items (figure e-1). This effect can be attributed mainly to pneumonia rather than to postoperative wound infections, because the rate of wound infections is low in this study. Notably, ubiquitous urinary 
A

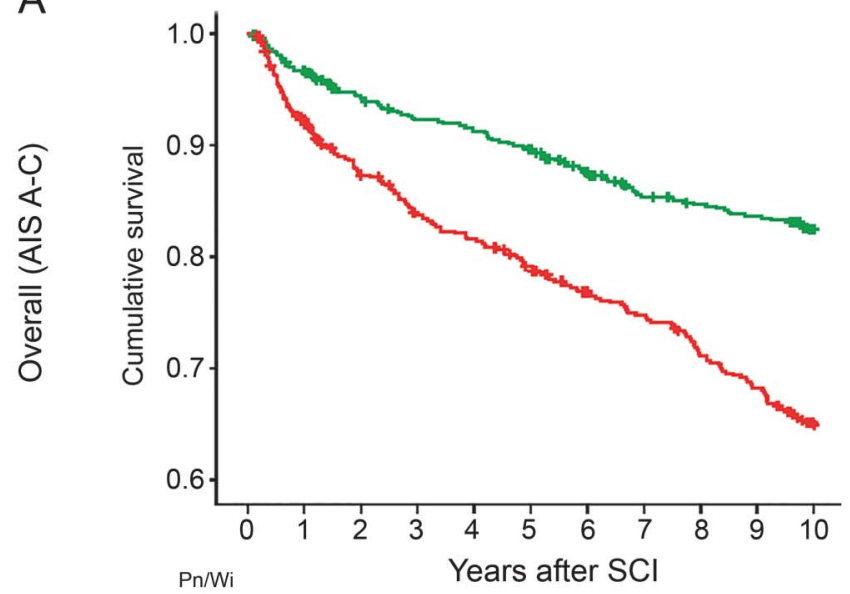

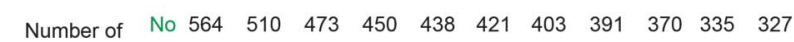

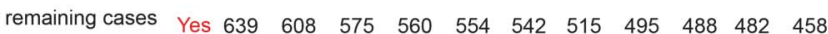

B

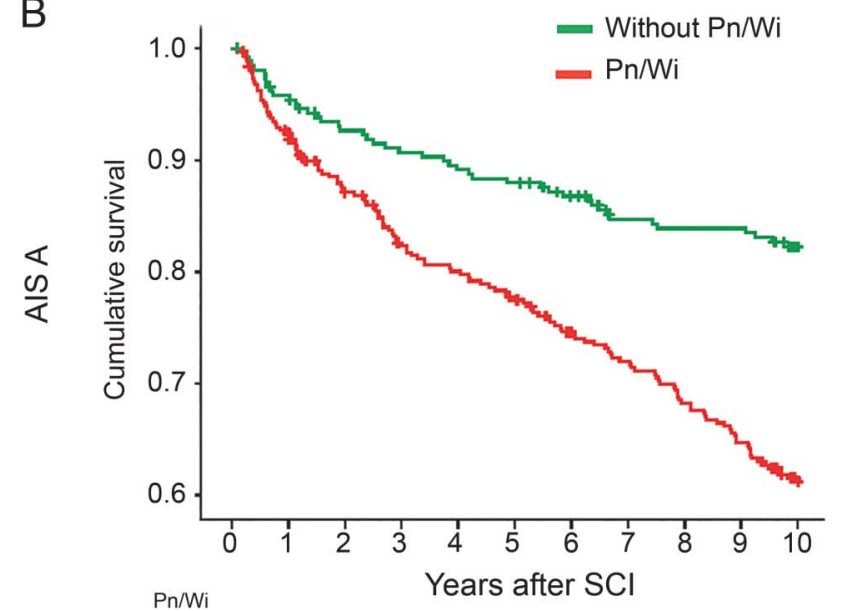

$\begin{array}{lllllllllllll}\text { Number of } & \text { No } & 261 & 248 & 237 & 232 & 228 & 225 & 215 & 205 & 203 & 203 & 189\end{array}$

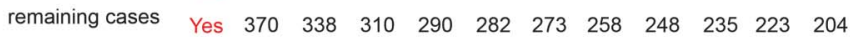

C

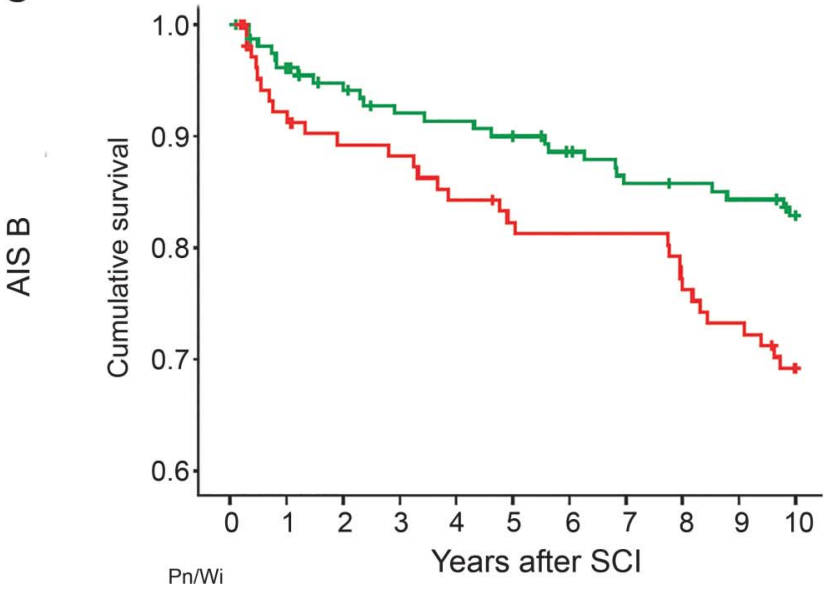

$\begin{array}{llllllllllll}\text { Number of } & \text { No } 157 & 147 & 140 & 134 & 133 & 131 & 126 & 121 & 120 & 118 & 113\end{array}$

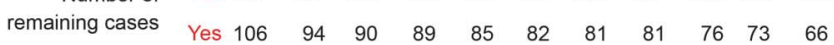
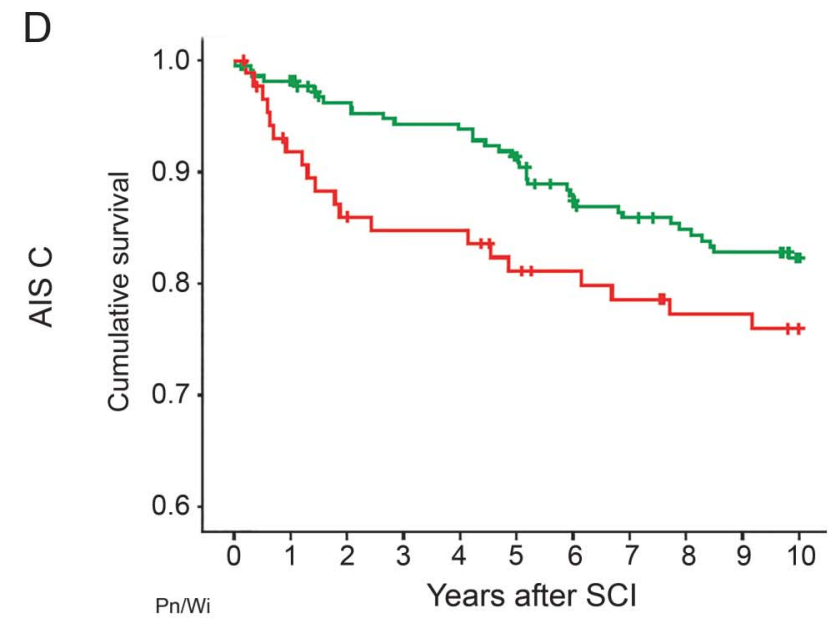

$\begin{array}{llllllllllll}\text { Number of } & \text { No } 221 & 214 & 198 & 194 & 193 & 186 & 174 & 169 & 165 & 161 & 156\end{array}$ $\begin{array}{lllllllllllll}\text { remaining cases } & \text { Yes } & 88 & 78 & 73 & 71 & 71 & 66 & 64 & 62 & 59 & 59 & 57\end{array}$

Kaplan-Meier curves indicate an increased cumulative mortality for patients with pneumonia or postoperative wound infection (Pn/Wi) in the overall population (A) and subsequent stratification for injury severity (B-D) during the 5-year follow-up. Sample size: number of events of death, number of patients. (A) Total sample (American Spinal Cord Injury Association impairment scale [AIS] A-C): $n=294, n=1,203$; (B) AIS A: $n=182, n=631 ;$ (C) AIS B: $n=56, n=$ 263; (D) AIS C: $n=56, n=309$. $S C l=$ spinal cord injury.

tract infections are not encoded in the NSCID and thus could not be taken into account. The observed effects might be even stronger if all infections could be excluded from the group without $\mathrm{Pn} / \mathrm{Wi}$.

Hospital-acquired $\mathrm{Pn} / \mathrm{Wi}$ have extended effects beyond neurologic impairment after cervical SCI and are potent denominators associated with poor longterm recovery of physical independence. Consequently, $\mathrm{Pn} / \mathrm{Wi}$ qualify as targetable disease-modifying factors after SCI. Moreover, hospital-acquired $\mathrm{Pn} / \mathrm{Wi}$ during the first months after SCI are associated with longterm mortality thereafter independent of baseline characteristics such as age, lesion level, and severity.

Considering patients with SCI to be at risk for developing infections encourages a rethinking of treatment algorithms to improve medical care. The development of early diagnostic tools will be instrumental to inform effective, individualized preventive therapeutic strategies (precision medicine). ${ }^{34}$ It is warranted to extend from preventive antibiotic treatment regimens as being ineffective in reducing infection rates or improving outcome after ischemic CNS injury. ${ }^{35-37}$ We provide evidence for considering infections as rehabilitation confounders ${ }^{38}$ of relevance for patient stratification in clinical trials and the protection of endogenous recovery potential after SCI.

\section{AUTHOR CONTRIBUTIONS}

Marcel A. Kopp: study concept and design, statistical analysis and interpretation of data, drafting of the manuscript, critical revision of the manuscript for intellectual content. Ralf Watzlawick: study concept and design, statistical analysis and interpretation of data, drafting of the manuscript. Peter Martus: statistical analysis and interpretation of data, 
critical revision of the manuscript for intellectual content. Vieri Failli: study concept and design, critical revision of the manuscript for intellectual content. Felix W. Finkenstaedt: statistical analysis and interpretation of data, critical revision of the manuscript for intellectual content. Yuying Chen: study concept and design, acquisition of data and database clearing, critical revision of the manuscript for intellectual content. Michael J. DeVivo: study concept and design, acquisition of data and database clearing, critical revision of the manuscript for intellectual content. Ulrich Dirnagl: study concept and design, critical revision of the manuscript for intellectual content. Jan M. Schwab: study concept and design, critical revision of the manuscript for intellectual content.

\section{STUDY FUNDING}

The study received funding from the Wings for Life Spinal Cord Research Foundation, Austria (grant WfL-DE-006/12), and the National Institute on Disability, Independent Living, and Rehabilitation Research (NIDILRR; grant 90DP0011 and 90SI50200100). NIDILRR is a Center within the Administration for Community Living, US Department of Health and Human Services.

\section{DISCLOSURE}

M. Kopp, R. Watzlawick, P. Martus, V. Failli, F. Finkenstaedt, Y. Chen, M. DeVivo, and U. Dirnagl report no disclosures relevant to the manuscript. J. Schwab received funding from the W.E. Hunt and C.M. Miller Endowment and is a Discovery Theme Initiative Scholar (OSU). Go to Neurology.org for full disclosures.

Received March 20, 2016. Accepted in final form December 9, 2016.

\section{REFERENCES}

1. Dietz V, Curt A. Translating preclinical approaches into human application. Handb Clin Neurol 2012;109:399-409.

2. Siddiqui AM, Khazaei M, Fehlings MG. Translating mechanisms of neuroprotection, regeneration, and repair to treatment of spinal cord injury. Prog Brain Res 2015; 218:15-54.

3. Curt A, Van Hedel HJ, Klaus D, Dietz V, EM-SCI Study Group. Recovery from a spinal cord injury: significance of compensation, neural plasticity, and repair. J Neurotrauma 2008;25:677-685.

4. Fawcett JW, Curt A, Steeves JD, et al. Guidelines for the conduct of clinical trials for spinal cord injury as developed by the ICCP panel: spontaneous recovery after spinal cord injury and statistical power needed for therapeutic clinical trials. Spinal Cord 2007;45:190-205.

5. Berlly M, Shem K. Respiratory management during the first five days after spinal cord injury. J Spinal Cord Med 2007;30:309-318.

6. Grossman RG, Frankowski RF, Burau KD, et al. Incidence and severity of acute complications after spinal cord injury. J Neurosurg Spine 2012;17:119-128.

7. DeVivo MJ, Krause JS, Lammertse DP. Recent trends in mortality and causes of death among persons with spinal cord injury. Arch Phys Med Rehabil 1999;80:1411-1419.

8. Shavelle RM, DeVivo MJ, Brooks JC, Strauss DJ, Paculdo DR. Improvements in long-term survival after spinal cord injury? Arch Phys Med Rehabil 2015;96:645-651.

9. Furlan JC, Krassioukov AV, Fehlings MG. Hematologic abnormalities within the first week after acute isolated traumatic cervical spinal cord injury: a case-control cohort study. Spine 2006;31:2674-2683.

10. Held KS, Steward O, Blanc C, Lane TE. Impaired immune responses following spinal cord injury lead to reduced ability to control viral infection. Exp Neurol 2010;226:242-253.

11. Lucin KM, Sanders VM, Jones TB, Malarkey WB, Popovich PG. Impaired antibody synthesis after spinal cord injury is level dependent and is due to sympathetic nervous system dysregulation. Exp Neurol 2007;207:75-84.

12. Meisel C, Schwab JM, Prass K, Meisel A, Dirnagl U. Central nervous system injury-induced immune deficiency syndrome. Nat Rev Neurosci 2005;6:775-786.

13. Riegger T, Conrad S, Liu K, Schluesener HJ, Adibzahdeh M, Schwab JM. Spinal cord injury-induced immune depression syndrome (SCI-IDS). Eur J Neurosci 2007;25:1743-1747.

14. Riegger T, Conrad S, Schluesener HJ, et al. Immune depression syndrome following human spinal cord injury (SCI): a pilot study. Neuroscience 2009;158:1194-1199.

15. Riegger T, Schluesener HJ, Conrad S, et al. Hematologic Cellular Inflammatory Response Following Human Spinal Cord Injury: 48th Annual Meeting of the German Society for Neuropathology and Neuroanatomy. Berlin: Acta Neuropathol; 2003:392.

16. Schwab JM, Zhang Y, Kopp MA, Brommer B, Popovich PG. The paradox of chronic neuroinflammation, systemic immune suppression, autoimmunity after traumatic chronic spinal cord injury. Exp Neurol 2014;258:121-129.

17. Vega JL, Ganea D, Jonakait GM. Acute down-regulation of antibody production following spinal cord injury: role of systemic catecholamines. J Neuropathol Exp Neurol 2003;62:848-854.

18. Brommer B, Engel O, Kopp MA, et al. Spinal cord injuryinduced immune deficiency syndrome enhances infection susceptibility dependent on lesion level. Brain 2016;139:692-707.

19. Failli V, Kopp MA, Gericke C, et al. Functional neurological recovery after spinal cord injury is impaired in patients with infections. Brain 2012;135:3238-3250.

20. DeVivo MJ, Go BK, Jackson AB. Overview of the national spinal cord injury statistical center database. J Spinal Cord Med 2002;25:335-338.

21. Marino RJ, Ditunno JF Jr, Donovan WH, Maynard F Jr. Neurologic recovery after traumatic spinal cord injury: data from the Model Spinal Cord Injury Systems. Arch Phys Med Rehabil 1999;80:1391-1396.

22. Richards JS, Go BK, Rutt RD, Lazarus PB. The National Spinal Cord Injury Collaborative Database. In: Spinal Cord Injury: Clinical Outcomes from the Model Systems. Gaithersburg, MD: Aspen Publishers; 1995:10-20.

23. Stover SL, DeVivo MJ, Go BK. History, implementation, and current status of the national spinal cord injury database. Arch Phys Med Rehabil 1999;80:1365-1371.

24. Galloway RV, Granger CV, Karmarkar AM, et al. The Uniform Data System for Medical Rehabilitation: report of patients with debility discharged from inpatient rehabilitation programs in 2000-2010. Am J Phys Med Rehabil 2013;92:14-27.

25. van Hedel HJ, Dietz V, European Multicenter Study on Human Spinal Cord Injury Study Group. Walking during daily life can be validly and responsively assessed in subjects with a spinal cord injury. Neurorehabil Neural Repair 2009;23:117-124.

26. Anderson KD, Acuff ME, Arp BG, et al. United States (US) multi-center study to assess the validity and reliability of the Spinal Cord Independence Measure (SCIM III). Spinal Cord 2011;49:880-885.

27. Granger CV, Harper C, Duffey E. The FIM-SR (selfreport) is not the FIM instrument. Arch Phys Med Rehabil 2007;88:265-266; author reply 266-267.

28. Marino RJ, Barros T, Biering-Sorensen F, et al. International standards for neurological classification of spinal cord injury. J Spinal Cord Med 2003;26(suppl 1):S50-S56. 
29. Rubin DB, Schenker N. Multiple imputation in healthcare databases: an overview and some applications. Stat Med 1991;10:585-598.

30. van Buuren S. Flexible Imputation of Missing Data. Boca Raton, FL: Chapman \& Hall/CRC Press; 2012.

31. Wilson JR, Cadotte DW, Fehlings MG. Clinical predictors of neurological outcome, functional status, and survival after traumatic spinal cord injury: a systematic review. J Neurosurg Spine 2012;17:11-26.

32. Furlan JC, Fehlings MG, Tator CH, Davis AM. Motor and sensory assessment of patients in clinical trials for pharmacological therapy of acute spinal cord injury: psychometric properties of the ASIA Standards. J Neurotrauma 2008;25: 1273-1301.

33. Little RJ, Wang Y. Pattern-mixture models for multivariate incomplete data with covariates. Biometrics 1996;52: 98-111.

34. Kopp MA, Druschel C, Meisel C, et al. The SCIentinel study: prospective multicenter study to define the spinal cord injury-induced immune depression syndrome (SCIIDS): study protocol and interim feasibility data. BMC Neurol 2013;13:168.

35. Harms H, Prass K, Meisel C, et al. Preventive antibacterial therapy in acute ischemic stroke: a randomized controlled trial. PLoS One 2008;3:e2158.

36. Kalra L, Irshad S, Hodsoll J, et al. Prophylactic antibiotics after acute stroke for reducing pneumonia in patients with dysphagia (STROKE-INF): a prospective, clusterrandomised, open-label, masked endpoint, controlled clinical trial. Lancet 2015;386:1835-1844.

37. Westendorp WF, Vermeij JD, Zock E, et al. The Preventive Antibiotics in Stroke Study (PASS): a pragmatic randomised open-label masked endpoint clinical trial. Lancet 2015;385:1519-1526.

38. Scivoletto G, Bonavita J, Torre M, et al. Observational study of the effectiveness of spinal cord injury rehabilitation using the Spinal Cord Injury-Ability Realization Measurement Index. Spinal Cord 2016;54:467-472.

\section{Neurology MOC Prep Course-Now Available Online and In-person!}

Recertification approaching? Looking for a comprehensive review in key areas of clinical neurology? Prepare for the ABPN recertification exam in clinical neurology by attending a Neurology MOC Prep Course-now offered in both in-person and online formats to meet your preferred learning style. Learn more at $A A N$.com/view/MOCPrep.

\section{Visit the Neurology ${ }^{\circledR}$ Resident \& Fellow Website}

Click on Residents \& Fellows tab at Neurology.org.

Now offering:

- Neurology ${ }^{\circledR}$ Resident \& Fellow Editorial team information

- "Search by subcategory" option

- E-pearl of the Week

- RSS Feeds

- Direct links to Continuum ${ }^{\circledR}$, Career Planning, and AAN Resident \& Fellow pages

- Recently published Resident \& Fellow articles

- Podcast descriptions

(1:ment Find Neurology ${ }^{\circledR}$ Residents \& Fellows Section on Facebook: http://tinyurl.com/o8ahsys

twitter Follow Neurology ${ }^{\circledR}$ on Twitter: http://twitter.com/GreenJournal 